\title{
Using Velocity Anisotropy to Analyze Magnetohydrodynamic Turbulence in Giant Molecular Clouds
}

\author{
Alecio Madrid ${ }^{1}$, Audra Hernandez ${ }^{1}$, Blakesely Burkhart ${ }^{2}$, and Jonathan Tan ${ }^{3,4}$ \\ ${ }^{1}$ Department of Astronomy, University of Wisconsin-Madison, 475 North Charter Street, \\ Madison, WI 53706, USA \\ ${ }^{2}$ Department of Astronomy, Harvard University, 60 Garden Street, MS 46 Cambridge, MA 02138, \\ USA \\ ${ }^{3}$ Deptartment of Space, Earth, and Environment, Chalmers Universiy of Technology, SE-412 96 \\ Gothenburg, Sweden \\ ${ }^{4}$ Deparment of Astronomy, University of Virginia, Charlottesville, VA 22904, USA
}

\begin{abstract}
Theoretical studies have indicated that structure function (SF) analysis is likely a viable tool for studying interstellar magnetohydrodynamic (MHD) turbulence. Turbulence plays a critical role in important galactic processes including the structure and evolution of giant molecular clouds (GMCs) as well as the formation of sub-structures known to spawn stellar progenitors. This study investigates the limitations of SF analysis as a tool for gauging MHD turbulence in GMCs. There exist many different methods of extracting GMCs from survey data, yet there is no known studies investigating their effects on SF accuracy. Despite the consensus in recent literature this study finds that SF analysis may have potential accuracy issues when its necessary to extract components from larger survey data.
\end{abstract}

\section{Introduction}

Giant Molecular Clouds (GMCs) are gaseous structures composed of cold, dense interstellar gas. Observations indicate large (GMC) scale virialization contrasted by disturbed, heirarichal substructuring at small (sub-GMC) scales (e.g. Hernandez \& Tan 2015). The interplay of gravity, turbulence, and magnetic energy is thought to govern GMC structural and dynamical properties (e.g. McKee \& Ostriker 2007). Gauging the turbulent properties of GMCs is crucial for advancing current knowledge of the processes driving the formation of galactic evolution and stellar progenitors.

Turbulence and magnetic fields are pervasive throughout the gaseous interstellar medium (ISM). Recent studies have indicated that the interaction of turbulent and magnetic energy can induce eddy alignment along local magnetic field lines. Magnetohydrodynamic (MHD) turbulence in GMCs is very difficult to directly measure, and our understanding of its role in the gaseous ISM comes almost exclusively from theoretical models and simulations (e.g. Cho \& Lazarian 2003, Burkhart et al. 2014, Otto et al. 2017).

Perhaps one of the most profound findings in recent literature (e.g. Esquivel \& Lazarian 2011, Burkhart et al. 2014, Otto et al. 2017, Heyer et al 2008) is that the anisotropic nature of eddy alignment within a strong MHD regime should be easily detectable via velocity field statistical analysis. These findings have led to the study of Structure Function (SF) analysis as an indirect (i.e. statistical) yet promising method for gaging MHD turbulence in the ISM. While these studies have undoubtedly been crucial for advancing our ability to study MHD turbulence in an observational setting. It is important to note that the majority of studies have investigated SF analysis from a broad theoretical framework with little attention to observational limitations such as missing data and noisey or irregularly shaped data sets. It is these limitations that we inestigate with the goal of applying SF analysis to GMC molecular line emission data. 
We organize this paper in the following manner. The physical properties of MHD turbulence and GMCs are discussed in $\S 2.1$ and $\S 2.2$ respectively. We give a brief overview of the MHD simulations in $\S 3$ followed by our methods in $\S 4$ and our results in $\S 5$. present our results in 4 and finish with a discussion of their implications, limitations and future work in $\S 6$.

\section{Magnetohydrodynamic Turbulence and Giant Molecular Clouds}

2.1. MHD turbulence While there is continued debate over the source and scale of energy injection, turbulence is an intrinsic property of interstellar gas. While classic Kolmogorov style hydrodynamic turbulence is driven isotopically the ionized nature of interstellar gas coupled with the ubiquitous magnetization of the ISM has the ability to induce highly anisotropic behavior in the form of MHD turbulence (Goldreich \& Sridhar 1995). While magnetic energy is constant across all scales, kinetic energy exhibits strong scale dependence (Cho \& Lazarian 2003). A profound finding was such that a sufficiently strong magnetic field (relative to the kinetic energy of the fluid) will confine parallel fluid oscillations. (Cho \& Vishniac 2000b, Maron \& Goldreich 2001, Cho, Lazarian \& Vishniac 2002a).

The Alfvén speed of a turbulent fluid is defined as $V_{a}=|\mathbf{B}| / \sqrt{4 \pi \rho}$, where $\mathbf{B}$ is the magnitude of the magnetic field vector and $\rho$ is the fluid density. The Alfvénic Mach Number $\left(M_{A}\right)$ expresses the ratio of magnetic energy to kinetic (i.e. turbulent) energy such that $M_{A}=V_{L} / V_{a}$, where $V_{L}$ is the turbulence injection velocity. All properties are averaged over the computational region. $M_{A}$ is of critical importance for a magnetically confined gas, indicating whether turbulent or magnetic energy is favored. MHD turbulence is partitioned into three qualitatively distinct regimes depending on which form of energy governs the fluid motion. The regimes are known as super-Alfvénic, trans-Alfvénic and sub-Alfvénic with $M_{A}>>1, M_{A} \approx 1$, and $M_{A}<<1$ respectively (e.g. Goldreich \& Sridhar 1995, Cho \& Lazarian 2003, Esquivel \& Lazarian 2011).

Another feature of MHD turbulence is the generation ofAlfvén waves which propagate along the magnetic field lines. Collisions of wave packets further exacerbate existing anisotropy while dissipating energy to smaller and smaller scales. This often results in significant energy imbalances leading to increased gas fragmentation especially at smaller scales (Cho \& Lazarian 2003).

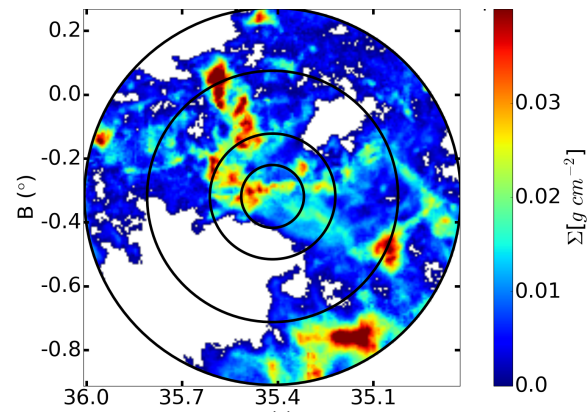

Figure 1: Integrated intensity map of GMC B from Hernandez \& Tan (2015)

these phenomena continues to be debated, but recent studies implicate the importance of MHD turbulence. While the injection source of interstellar MHD turbulence is not well-understood, there is consistent agreement that injection scales are much larger than typical GMC size (e.g. Burkhart et al. 2014, Murray 2011).

\section{MHD Simulations}

The data used in this study was obtained from two three-dimensional numerical simulations of isothermal compressible MHD turbulence with known physical parameters from Burkhart et al. (2014). We studied two different simulations, which we call L15 and L7, both with a resolution of $512^{3}$. Figure 2 shows the average column density for the L7 simulation integrated in the Z LOS. Both were run with large scale solenoidal forcing with a uniform background magnetic field and a fluctuating turbulent field for $t \approx 5$ crossing times in order to guarantee a fully developed energy cascade. 


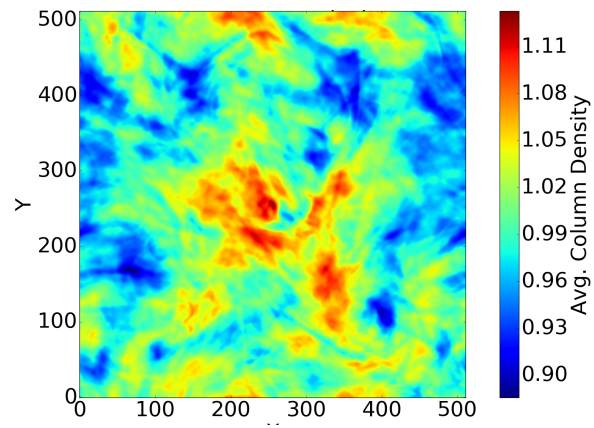

Figure 2: Integrated column density map for L7 Z LOS.

The simulations were given unitless magnetic field strengths such that $|\mathbf{B}|<1,|\mathbf{B}|=1,|\mathbf{B}|>1$ were considered to be super-Alfvénic, trans-Alfvénic and subAlfvénic respectively. Simulation L15 is considered to be trans-Alfvénic with $|\mathbf{B}| \approx 1$, while L7 is considered super-Alfvénic with $|\mathbf{B}| \approx 0.1$. In both simulations the global magnetic field points in the $\mathrm{x}$ direction, though the local magnetic field strength and direction within a given aperture may differ. For full simulation properties see Burkhart et al.(2014), Burkhart et al. (2009), Cho \& Lazarian (2003), or Kowal et al. (2007).

\section{Methods}

4.1. Structure Function Analysis Our primary tool in this study was two-dimensional real space SF analysis. The term real space is used to distinguish between the iterative SF algorithm used in this paper and the Fast Fourier Transform (FFT) method typically seen in current literature. Unlike the FFT method, our real space method allows for analysis of irregularly shaped data sets or those with missing or masked data by means of direct iterative computation. We define the two-dimensional real space SF in Equation 1.

$$
S F(\mathbf{R})=\frac{1}{N} \sum_{x=0}^{\operatorname{len}(x)} \sum_{y=0}^{\operatorname{len}(y)}\left(V_{\mathbf{R}}-V_{x y}\right)^{2} .
$$

$\mathbf{R}$ denotes a position vector on the SF map with integer components, $V_{x y}$ denotes the line of sight (LOS) velocity component at a given position (x,y), and $V_{\mathbf{R}}$ denotes the LOS velocity component at position $\mathbf{R} . V_{x y}$ and $V_{\mathbf{R}}$ are defined to be real valued pixels, with $\mathrm{N}$ defined as the total number of real valued pixels in the array.

4.2. Deriving magetic properties In order to measure the isotropic nature of the two-dimensional SF, we measure what has been termed the isotropy degree. The isotropy degree is defined as the ratio of values along the average SF major axis to the values along the average minor axis. The term average is used because it is calculated by sampling the isotropy direction of a range of SF isocontours.

In order to find the average major and minor axese, we calculate the average SF ellipse within a defined velocity interval $(\Delta V)$ of closed isocontours using a modified version of the fitEllipse (vanForeest 2012) Python module. We identify the maximum closed SF ellipse $(E)$ within \pm half of the turbulence injection space such that $\Delta V=\left[\frac{E}{10}, E\right]$. We then choose our smaple contours as $S=\left[\frac{E}{10}, \frac{2 E}{10}, \frac{3 E}{10}, \ldots, E\right]$. The isotropy degree can then be calculated using Equation 2.

$$
I(\mathbf{D})=\frac{S F_{m a j}(\mathbf{D})}{S F_{\min }(\mathbf{D})}
$$

D is defined as the radial distance from the SF origin.

4.3. Limitation analysis The non-isolated nature of GMCs, the dynamic nature of the ISM, and the presence of unrelated structures in a given velocity channel are just three examples of phenomena that complicate the process of extracting GMCs from survey data (Lee et al. 2016). Studies have found that despite the large variety of methods used for extracting GMCs, there is a notable consistency in their derived properties and scaling relations (Hennebelle \& Falgarone 2012). Given a GMC with known physical properties, SF analysis should show reasonable consitency when isolating the GMC structure from a larger data set.

4.3. Density Threshold Effects The motivation for the density threshold component of our study comes from what Hernandez \& Tan (2015) term connected extraction (CE). Using this method they define a cloud as a connected structure in position-position-velocity (PPV) space such that every voxel is above a minimum intensity threshold (specifically the GRS $5 \sigma_{r m s}$ noise level). For a detailed review of the CE method see Hernandez \& Tan (2015). Future studies will implement the CE method in PPV space, but for this study we only test the effects of a minimum 
density threshold in position-position space. Our goal is to determine whether varying the density threshold can induce discrepancies between the known physical properties and those derived from the SF.

We defined a range of minimum column density thresholds and study the effects of masking data below the specified density. We implemented the threshold masks on both simulations in all three axial directions. The masks were defined by $\mathrm{N}+(\mathrm{p} * \mathrm{~N})$ where $\mathrm{N}$ is the mean column density and $\mathrm{p}$ is a percentage. For both clouds we calculated the SF and isotropy degree for the original non-masked simulations as well as for $\mathrm{p}=10,12,14,16,18$, and $20 \%$.

4.3. Aperture effects The motivation for the aperture component of our study comes from what Hernandez \& Tan (2015) term simple extraction (SE). In the SE method, they define the GMC structure to be all gass within a defined velocity interval bounded by a circular boundary. They implement this method at multiple radii. We similarly consider gas within a circular aperature at three different radii (specifically 60,120 , and 180 pixels) centered at the simulation peak column density. We again do this for both simulations in all three axial directions. For the full density threshold and aperture trials please see Appendix A.

\section{Results}

5.1. Density threshold analysis Figure 3 shows the results from applying minimum density thresholds to both simulations. At each density threshold we measure the local magnetic field, the average isotropy degree, and the absolute alighment between the SF major axis and the angle of the local magnetic field vector (i.e. $0^{\circ} \leq \theta_{B}-\theta_{m a j} \leq$ $90^{\circ}$ ).

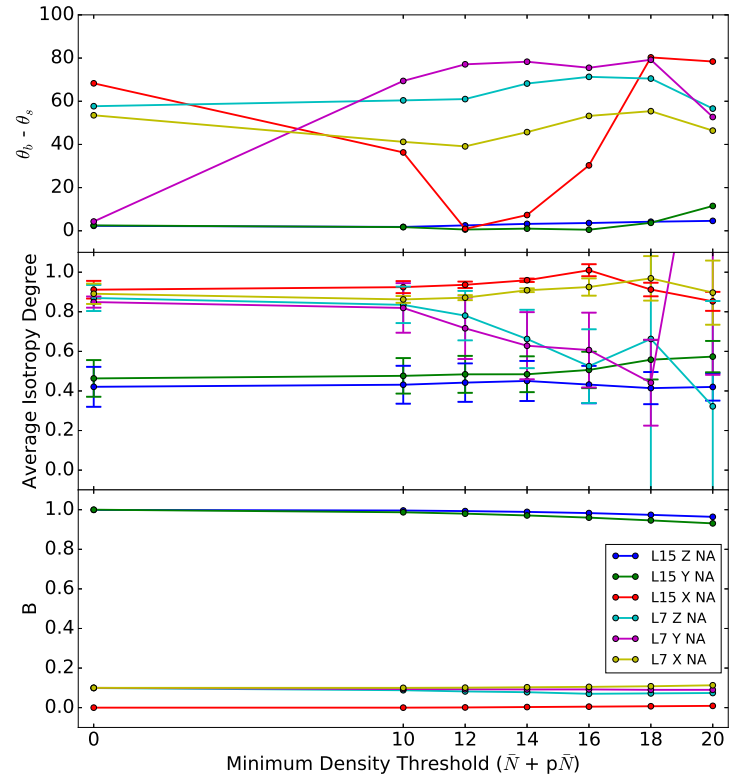

Figure 3: L7/L15 density threshold analysis. Top: Absolute difference between SF angle and B field angle as a function of density threshold. Middle: Average isotropy degree as a function of density threshold. Isotropy degree is measured as a function of separation, so it is necessary to include error when taking the average. Bottom: Magnetic field strength as a function of density threshold, calculated as the magnitude of the average magnetic field vector.
5.1. Magnetic field strength The bottom of Figure 3 displays magnetic field measurements at each density threshold. All three directions for both clouds show nearly constant magnetic field strength at every threshold. In the L15 Y and Z LOS we measure magnetic field strenghts consistent with a trans-Alfvénic regime $(|\mathbf{B}| \approx$ 1.0). In the L15 X LOS and the L7 simulation we note similar consitency albeit with magnetic field strengths consistent with a super-Alfvénic regime $(|\mathbf{B}|<<1.0)$

5.1. Isotropy degree We first note the large errors in the 18 and $20 \%$ threshold and do not include either case in our analysis. We believe that because of the large reduction in valid pixels there is no longer sufficient data to compute a valid statistical average. While this does demonstrate a SF limitation, it has been addressed in the current literature and we do not further pursue the issue here.

Row two of Figure 3 shows the isotropy degree for each trial as a function of density threshold. The isotropy degree for both the $\mathrm{Z}$ and Y LOS for L15 were found to be between 0.4 and 0.5 at every density threshold. Given the measured magnetic field strengh these results are consistent with what is predicted by the current literature. The X LOS for L15 and L7 both have isotropy degrees above 0.8 at every threshold, also consistent with what is predicted by the current literature. The isotropy degree in the L7 Z and the L7 Y LOS are not consistent with the expected results and provide cause for concern. Despite a consistently weak magnetic field, the isotropy degree 
decreases consistently as the threshold for both simulations is increased. Based on the current literature these measurements would indicate the presence of a much stronger magnetic field than that which is present. While further investigation is necessary to understand the underlying cause of the observed anisotropy, it likely that it is not caused by the local magnetic environment.

5.1. Magnetic field alignment Row one of Figure 3 shows the alignment of the average SF major axis with the magnetic field as a function of density threshold. In the L15 Y and Z LOS we observe consistent alignment between the SF angle and magnetic field angle at every threshold. In the super-Alfvénic data we observe erratic behavior indicating generally inconsistent alignment between the SF major axis and the magnetic field direction. We beleive the decrease in alignment at the $20 \%$ threshold for the L15 Y LOS is likely due to the fragmentation of the gas structure.

5.2. Aperture analysis For the aperture analysis we plot magnetic field strength, average isotropy degree and magnetic field alignment as a function of aperture size. Similar to the SE method from Hernandez \& Tan (2015) we include all data within circular apertures at 60, 120, and 180 pixel radii. In Figure 4 we include the full simulation for reference, denoted as 256 , but we do not place a circular aperture over the velocity centroid map.

5.2. Magnetic field strength The bottom panel of Figure 4 shows our magnetic field measurements as a function of aperture size. As in the density trial, we measure nominal change in in the local magnetic field strength for all cases.

5.2. Isotropy degree The middle panel in Figure 4 shows the average isotropy degree measured within each aperture. With the exception of L15 Y every case shows a decrease in the measured isotropy degre upon application of a 180 or 120 pixel aperture. Upon application of a 60 pixel aperture we observe an increase in isotropy in all three L7 LOS as well as in the L15

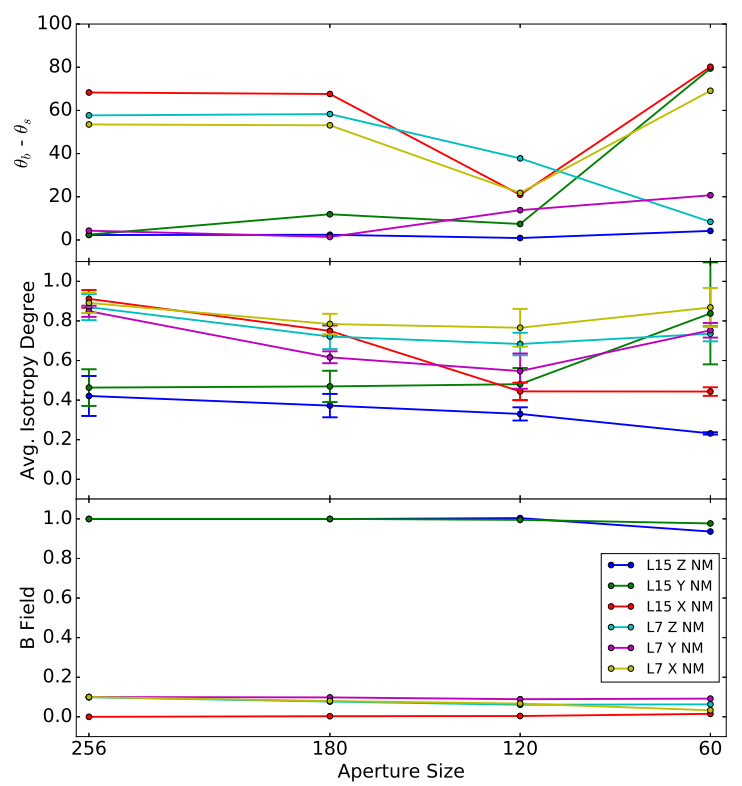

Figure 4: Same as Figure 3, but here we plot everything as a function of aperture size.
5.2. Magnetic field alignment Figure 4 panel one shows the absolute alignment between the average SF ellipse major axis and the magnetic field direction within each aperture. We observe strong alignment in L15 Z and L7 Y regardless of aperture size. We observe similar behavior for the L15 Y LOS in every case except for the 60 pixel aperture. L7 Z and X and L15 X all show generally weak alignment except for the sharp increase within the 120 pixel aperture.

\section{Discussion and Summary}

We performed an in-depth study of the observational limitations of SF analysis using MHD molecular gas simulations from two different Alfvénic regimes (Burkhart et al. 2014). We investigated the errors induced by different methods of GMC extraction, specifically SE and CE from Hernadez \& Tan (2015). We extracted simulated GMCs by first using a minimum density threshold followed by means of an induced aperture. The most significant finding is that even though we observed nominal changes in the magnetic energy there was significant variability of the isotropy degrees within the super-Alfvénic regimes.

In all simulations aside from the $\mathrm{R}=60$ aperture and the $20 \%$ density threshold, all of the trans-Alfvénic regimes returned isotropy degree and magnectic field alignments consistent with the expected results. Average isotropy degree 
measurements did not deviate significantly from 0.5 and alignment stayed consistently below $10^{\circ}$. As we noted in a number of the trials, the average isotropy degree in both the $\mathrm{R}=60$ aperture and $20 \%$ density threshold have very large errors which we beleive to be statistical in nature as a result of insufficient data.

In contrast, the super-Alfvénic regimes showed significant deviation from the expected results. We found that even if we dont consider cases with relatively large error measurements, both extraction methods lead to instances of low isotropy measurements within a super-Alfvénic regime. In the density threshold trial we found that at 14 and $16 \% \mathrm{~L} 7$ $\mathrm{Y}$ and $\mathrm{Z}$ both had isotropy degrees much lower than the expected values. We observed similar results in all five superAlfvénic trials with $R=180$ or 120 pixel apertures. Sans magnetic field information, the consensus in current literature would indicate that these measurements were from a trans or sub-Alfvénic regime (e.g. Esquivel et al. 2015, Cho et al. 2002, Nestingen-Palm et al. 2017, Burkhart et al. 2014). Given that the properties are not trans or sub-Alfvénic but are in fact super-Alfvénic indicates a potential limitation of SF analysis.

In summary, our results indicate that GMC extraction methods have the potential to significantly reduce the accuracy of SF results. We believe that the current understanding of SF analysis is promising, but presents notable issues when extracting components from larger data sets. We observe issues of both accuracy and robustness that we beleive require further study before this method can be accurately applied to molecular line emission data. Future work will focus on understanding the physical and statistical implications of the observed discrepancies as well as developing a framework for observational use with the goal of accurately apply our findings to GMC molecular line emission data.

\section{Acknowledgments}

A.M. acknowledges support from Wisconsin Space Grant Consortium Undergraduate Research Fellowship, support from the University of Wisconsin-Madison Hilldale Research Fellowship, as well as support from grants awarded to Audra Hernandez from the National Science Foundation. We also thank Christopher Herron for assistance in developing the two-dimensional real space structure function. This publication makes use of molecular line data from the Boston University-FCRAO Galactic Ring Survey (GRS). The GRS is a joint project of Boston University and Five College Radio Astronomy Observatory, funded by the National Science Foundation under grants AST-9800334, AST-0098562, AST-0100793, AST-0228993, \& AST-0507657.

\section{References}

Burkhart, B., Falceta-Gon 0€4alves, D., Kowal, G., \& Lazarian, A. 2009, ApJ, 693, 250

Burkhart, B., Lazarian, A., Le0€0o, I. C., de Medeiros, J. R., \& Esquivel, A. 2014, ApJ, 790, 130

Cho, J., \& Lazarian, A. 2003, MNRAS, 345, 325

Cho, J., Lazarian, A., \& Vishniaz, E.T. 2002, ApJ, 345, 325

Cho J., Vishniac E. 2000a, ApJ, 538, 217

Cho J., Vishniac E. 2000b, ApJ, 539, 273

Esquivel, A., \& Lazarian, A. 2005, ApJ, 631, 320

Esquivel, A., \& Lazarian, A. 2011, ApJ, 740, 117

Eqquivel, A., Lazarian, A., \& Pogosyan, D. 2015, ApJ, 814, 77

Garcła, P., Bronfman, Nyman, L., Dame, T.M., \& Luna, A. 2014, ApJS, 212,2

Goldreich P., Sridhar S. 1995, APJ, 438, 763

Hennebelle, P., \& Falgarone, E. 2012, A\&ARv, 20, 55

Heyer, M., Gong, H., Ostriker, E., \& Brunt, C. 2008, ApJ, 680, 420

Jackson, J. M., Rathborne, J. M., Shah, R. Y., et al. 2006 ApJ 163, 145

Kowal, G., Lazarian, A., \& Beresnyak, A. 2007 ApJ, 658, 423

Hernandez, A. K. \& Tan J. C. 2015, ApJ, 809, 154

Lee, E. J., Miville-Deschnes, M-A., Murray, N. W. 2016 ApJ, 833, 229

Lada, C. J., \& Lada, E. A. 2003, ARA\&A, 41, 57

Maron J., Goldreich P. 2001, ApJ, 554, 1175

McKee, C. F., \& Ostriker, E. C. 2007 ARA\&A, 45, 565

Nestingen-Palm, D., Stanimoirovi04, S., Gonzlez-Casanova, F. D., Babler, .B, Jameson, K., Bolatto, A. 2017, ApJ, 845,1

Murray, N. 2011, ApJ, 729, 133 
Otto, F., Ji, W., \& Li, H. 2017 ApJ 836, 95

VanForeest, N. 2012, fitEllipse.py

\section{Appendix A}


Figure 5: Column 1: Column denisty map L7 Z. Column 2: Velocity centroid map L7 Z. Column 2: Structure fuction map L7 Z. Row 1: 60 pixel aperture. Row 2: 120 pixel aperture. Row 3: 180 pixel aperture. Row 4: 10\% density threshold mask. Row 5: $12 \%$ density threshold mask. Row 6 : $14 \%$ density threshold mask. Row 7: $16 \%$ density threshold mask. Row 8 : 18\% density threshold mask. Row 9: $20 \%$ density threshold mask
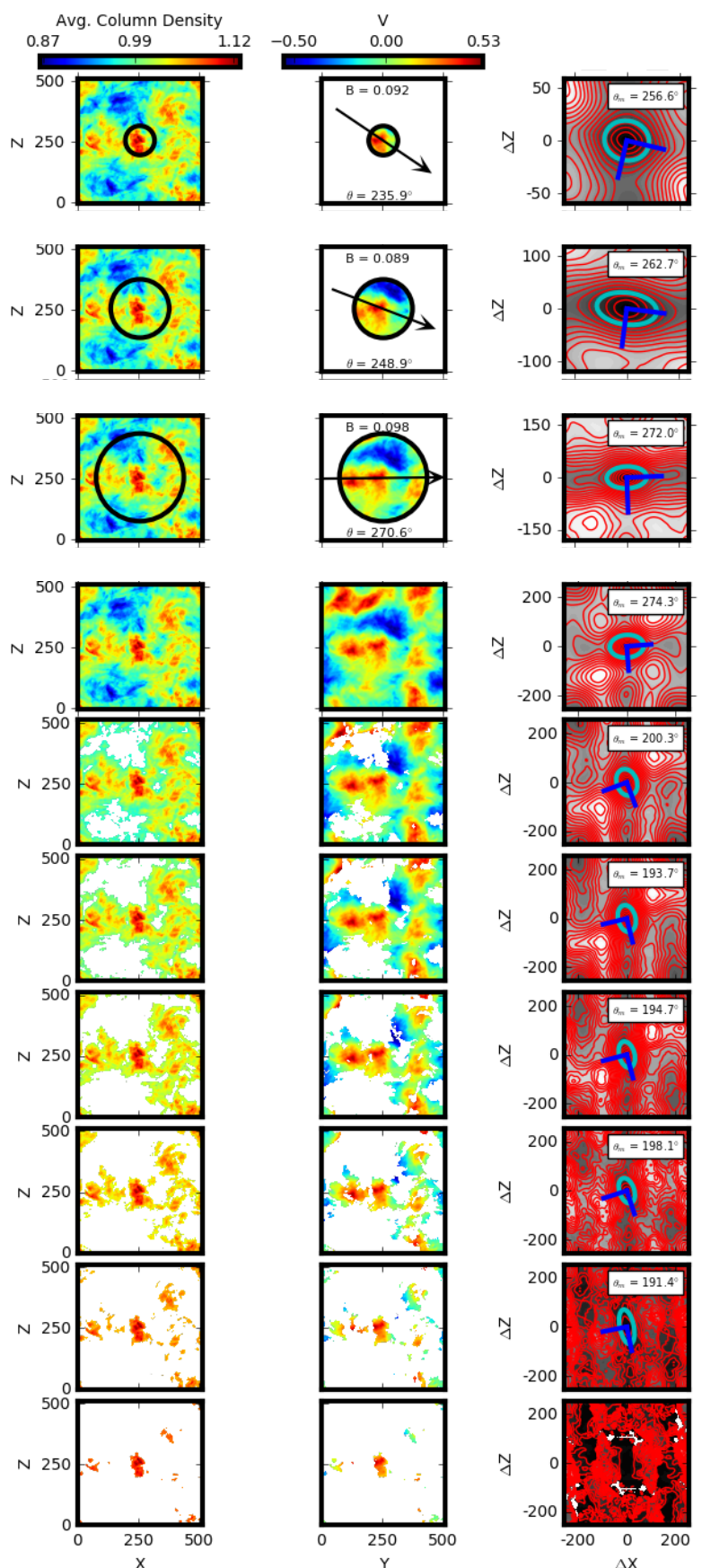

Figure 6: Same as Figure 5, but here we show L7 Y. 

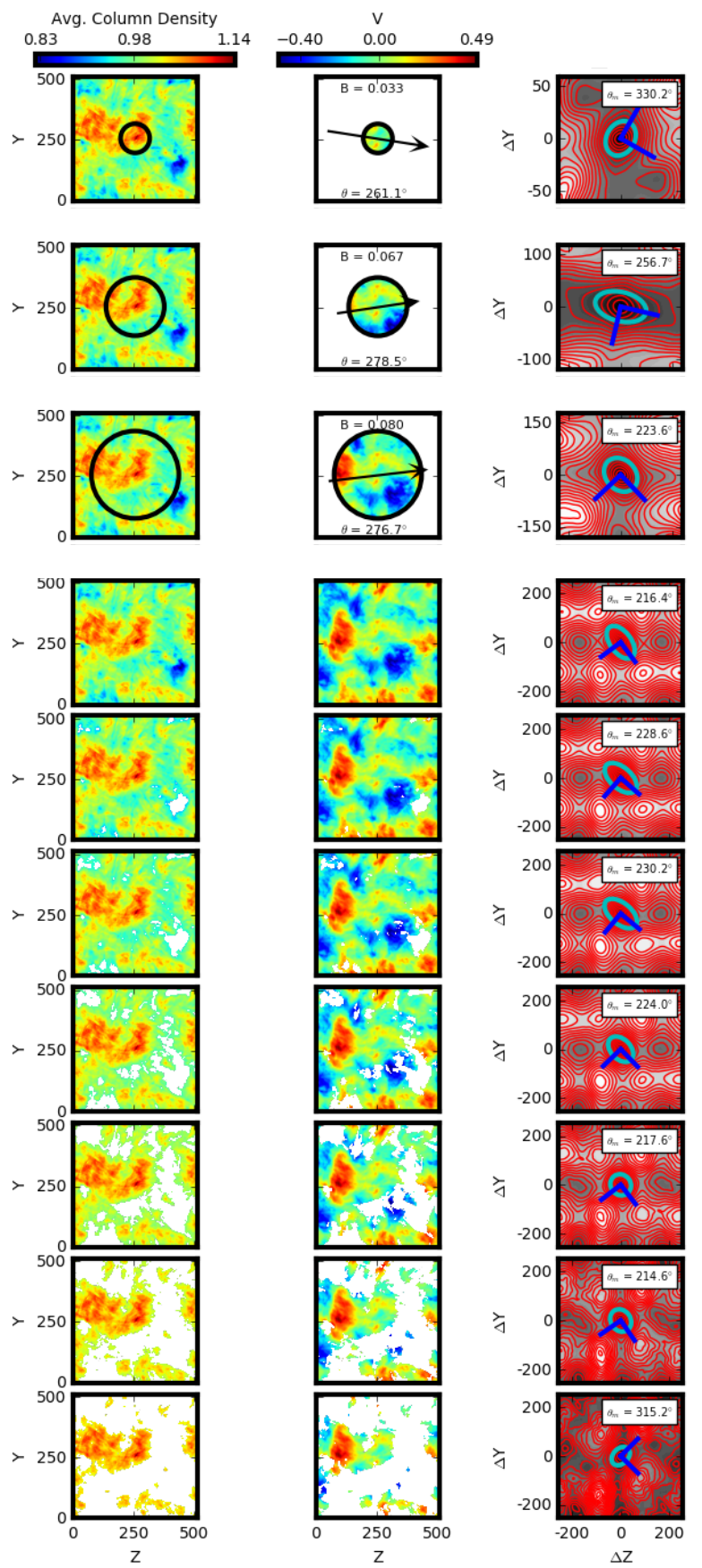

Figure 7: Same as Figure 5, but here we show L7 X.
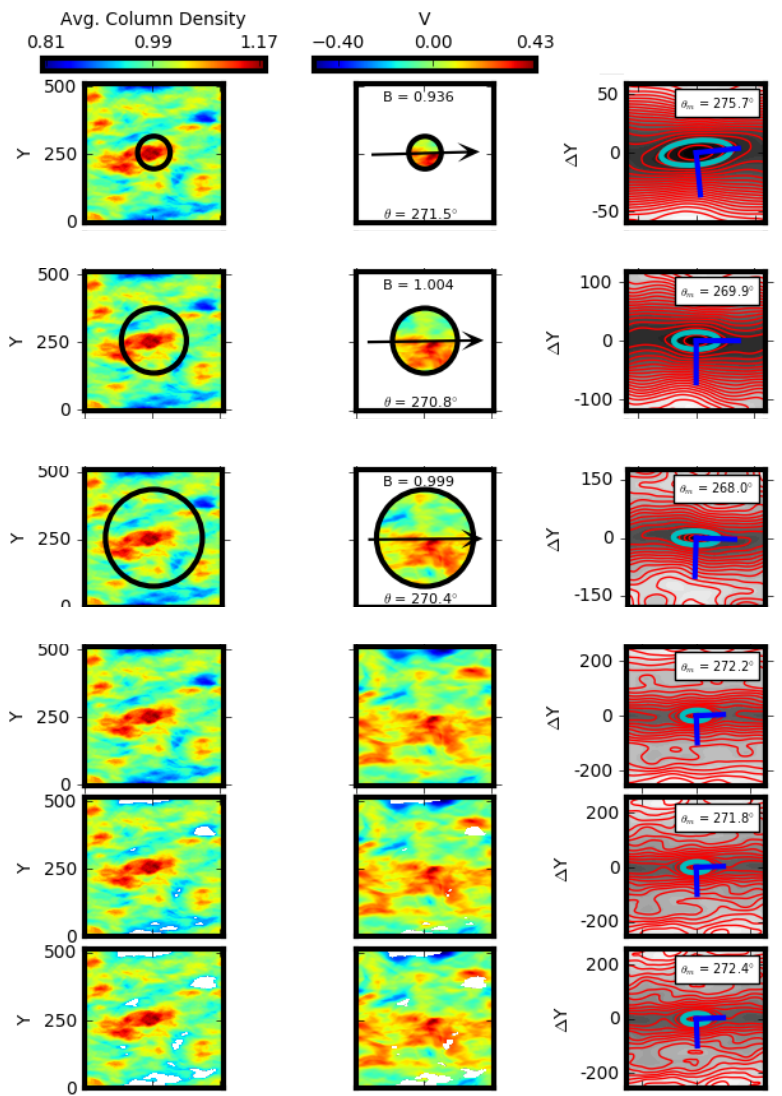

¿1
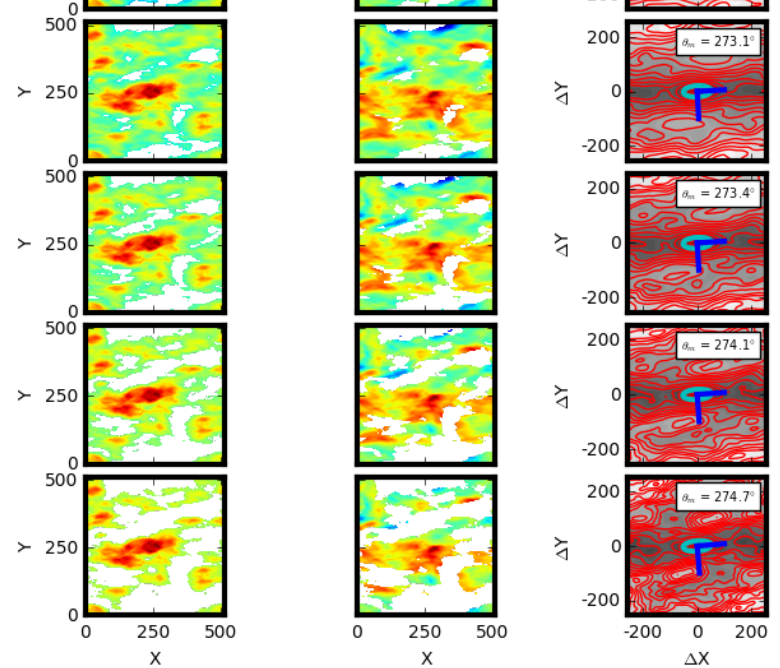

Figure 8: Same as Figure 5, but here we show L15 Z. 

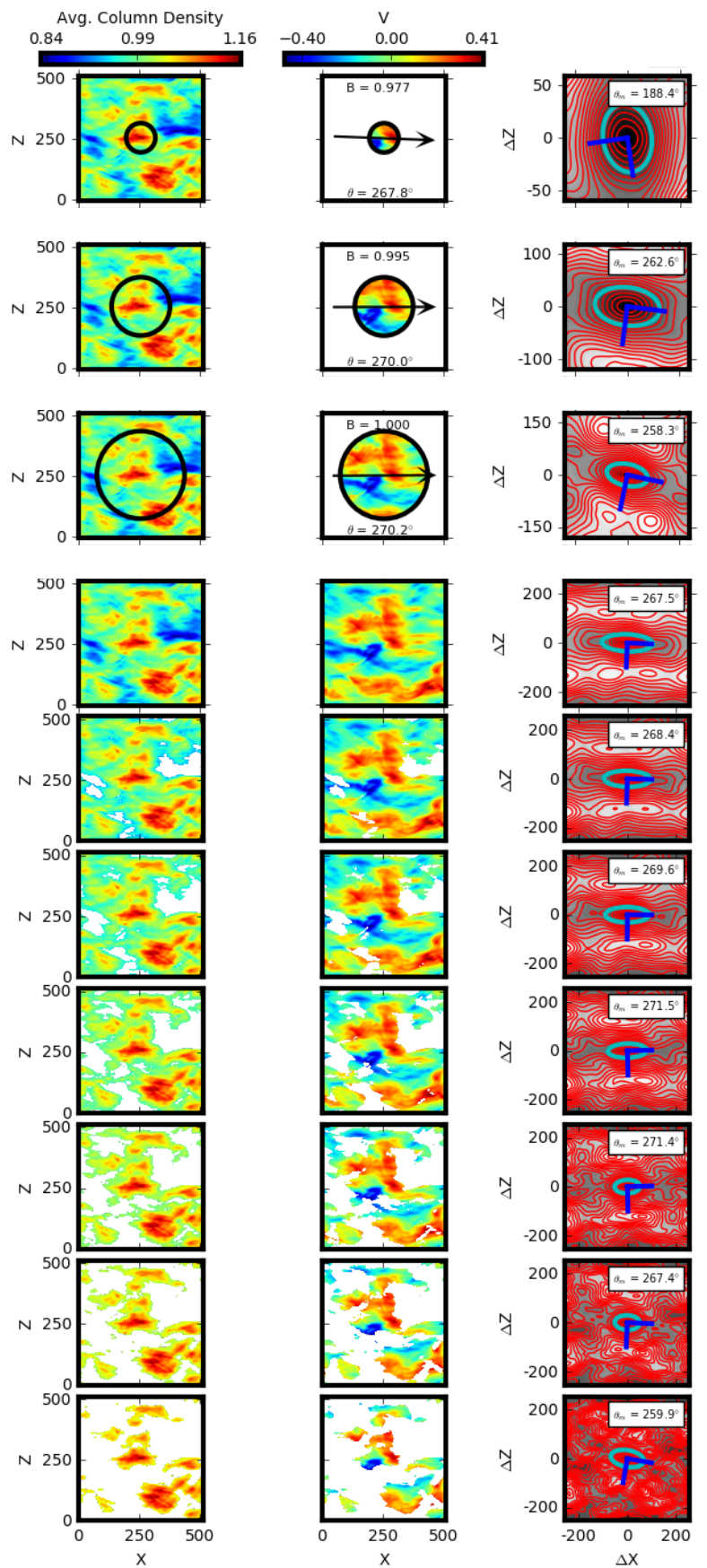

Figure 9: Same as Figure 5, but here we show L15 Y.
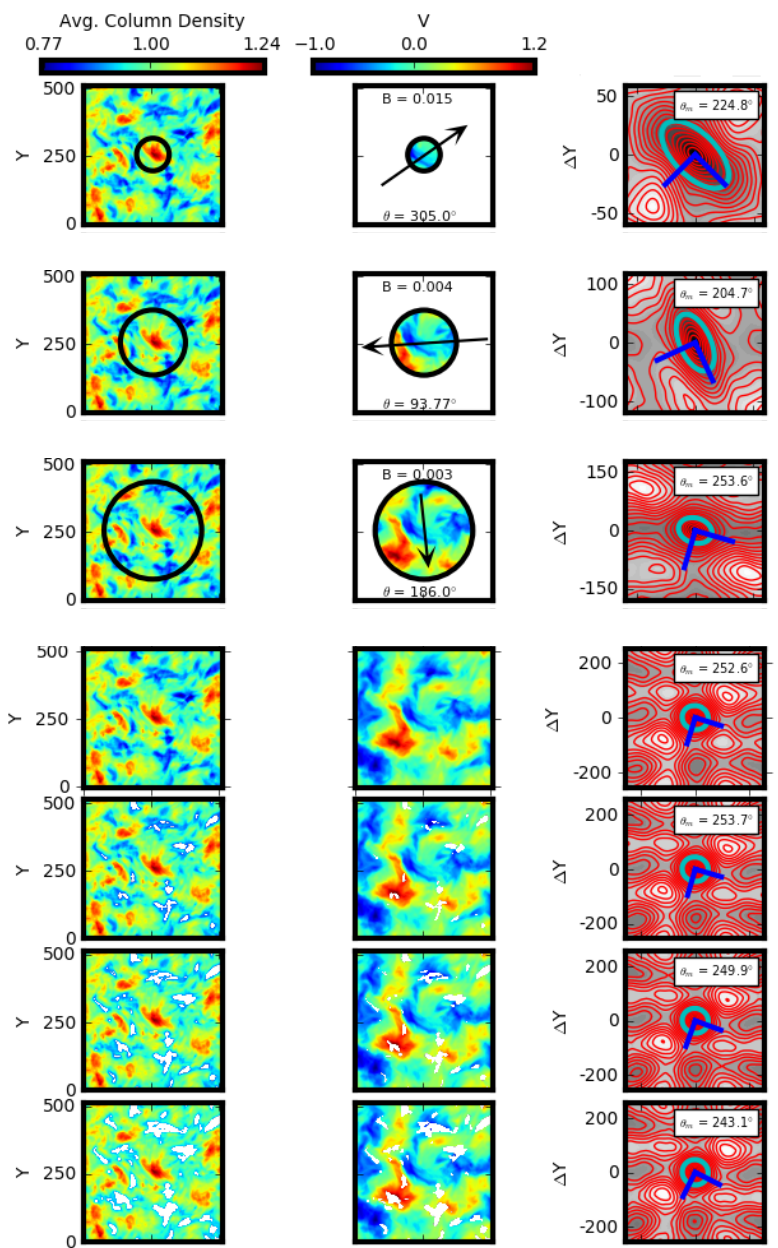

-200 All
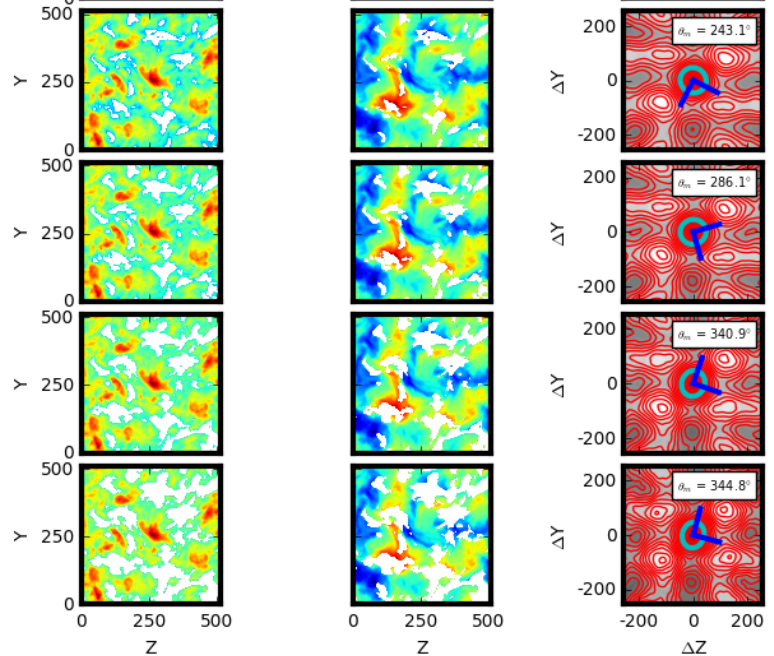

Figure 10: Same as Figure 5, but here we show L15 X. 